\title{
Right-handed sneutrinos as curvatons
}

\author{
John McDonald* \\ Department of Mathematical Sciences, University of Liverpool, Liverpool L69 3BX, England
}

(Received 13 March 2003; published 8 August 2003)

\begin{abstract}
We consider the possibility that a right-handed sneutrino can serve as the source of energy density perturbations leading to structure formation in cosmology. The cosmological evolution of a coherently oscillating condensate of right-handed sneutrinos is studied for the case where reheating after inflation is due to perturbative inflaton decays. For the case of Dirac neutrinos, it is shown that some suppression of Planck scalesuppressed corrections to the right-handed neutrino superpotential is necessary in order to have sufficiently late decay of the right-handed sneutrinos. $c H^{2}$ corrections to the sneutrino mass squared term must also be suppressed during inflation $(|c| \lesssim 0.1)$, in which case, depending on the magnitude of $|c|$ during inflation, a significantly blue (if $c>0$ ) or red (if $c<0$ ) perturbation spectrum is possible. $R$ parity must also be broken in order to ensure that the Universe is not overclosed by the lightest supersymmetric particles from the late decay (at temperatures $1-10 \mathrm{MeV}$ ) of the right-handed sneutrino condensate. The resulting expansion rate during inflation can be significantly smaller than in conventional supersymmetric inflation models (as low as $10^{6} \mathrm{GeV}$ is possible). For the case of Majorana neutrinos, a more severe suppression of Planck-suppressed superpotential corrections is required. In addition, the Majorana sneutrino condensate is likely to be thermalized before it can dominate the energy density, which would exclude the Majorana right-handed sneutrino as a curvaton.

DOI: 10.1103/PhysRevD.68.043505

PACS number(s): $98.80 . \mathrm{Cq}$
\end{abstract}

\section{INTRODUCTION}

The observation of neutrino masses and mass splittings, via solar and atmospheric neutrinos [1], strongly suggests the existence of right-handed $(\mathrm{RH})$ neutrinos. In extensions of the minimal supersymmetric standard model (MSSM) [2] which can accommodate neutrino masses we therefore expect to have right-handed sneutrinos. In the cosmology of the MSSM and its extensions, Bose condensates of scalar fields such as squarks and sleptons form naturally after inflation [4]. These may have important consequences for cosmology; for example, they allow for the possibility of baryogenesis and leptogenesis via the Affleck-Dine mechanism [4,5]. Since right-handed sneutrinos may also form condensates, it is important to consider in some detail the cosmological evolution of a RH sneutrino condensate.

Because of the weak coupling of the RH sneutrinos to the MSSM fields, a condensate of RH sneutrinos will be longlived and so may come to dominate the energy density of the Universe before it decays. The question of whether the RH sneutrino condensate can dominate the energy density of the Universe when it decays has recently acquired some importance. It has been noted $[6,7]$ that if a scalar dominates the energy density when it decays, and if that scalar is effectively massless during inflation, then quantum fluctuations of the scalar during inflation can in principle account for the primordial energy density perturbations leading to structure formation [6-8]. This has been labeled the curvaton scenario $[9,10]$. Should the curvaton be able to account for the density perturbations, the parameters of the inflation model would be more weakly constrained than in the conventional case where density perturbations arise from quantum fluctuations of the inflaton.

\footnotetext{
*Email address: mcdonald@amtp.liv.ac.uk
}

Thus if there exists a natural curvaton candidate such as the RH sneutrino, it is important to confirm or exclude that candidate as a curvaton. ${ }^{1}$ The main goal of this paper is to investigate the possibility of a $\mathrm{RH}$ sneutrino curvaton.

The masses of the RH sneutrinos and their coupling to the MSSM fields are determined by the model of neutrino masses, in particular whether they have Dirac masses or Majorana masses via a see-saw mechanism [1]. The Yukawa coupling of the RH sneutrino to the MSSM fields plays a fundamental role in determining the evolution of the $\mathrm{RH}$ sneutrino condensate, in particular its decay temperature, its effective mass from interacting with the background of inflaton decay products [13] and its rate of thermalization or scattering from the background.

Throughout this paper we will consider the simplest model for inflation and reheating, corresponding to a constant expansion rate during inflation followed by formation of a coherently oscillating inflaton condensate and reheating due to perturbative inflaton decays.

The paper is organized as follows. In Sec. II we discuss the cosmological environment due to perturbative inflaton decays. In Sec. III we consider the evolution of the RH sneutrino condensate in this environment. In Sec. IV we present our conclusions.

\section{COSMOLOGICAL BACKGROUND FROM PERTURBATIVE INFLATON DECAYS}

After inflation we consider the inflaton $S$ to have a mass $m_{S}$ and to be coherently oscillating about the minimum of its potential. The inflaton is assumed to decay into pairs of rela-

\footnotetext{
${ }^{1}$ Models have recently been proposed where the curvaton corresponds to an MSSM flat direction scalar [11] and an MSSM Higgs scalar [12].
} 
tivistic MSSM particles with initial energy $m_{S} / 2$. If the initial energy of the decay products is sufficiently large, the initial scattering rate of the inflaton decay products may be small enough that thermalization only occurs once the Universe has expanded sufficiently for the decay product scattering rate $\Gamma_{s c}$ to exceed the expansion rate $H$. There are therefore two possibilities.

(a) Instantaneous thermalization. The inflaton decay products thermalize immediately after decay. In this case there will be two epochs: (i) inflaton matter domination (IMD), where the energy density of the Universe is dominated by the coherently oscillating inflaton, and (ii) radiation domination (RD), defined to mean domination of the energy density of the Universe by relativistic inflaton decay products, not necessarily thermalized.

(b) Noninstantaneous thermalization. The relativistic inflaton decay products are unthermalized initially. In this case we will show that thermalization cannot occur during IMD and so must occur during RD. Therefore in this case the background will have three distinct epochs: (i) IMD, (ii) RD pre-thermalization and (iii) RD post-thermalization.

\section{A. Inflaton decay product thermalization}

Prior to thermalization there is a spectrum of decay products, ranging from redshifted products from the earliest decays (occurring at the end of inflation) to products from the most recent decays. Most of the energy density in decay products will come from the most recent decays. Following the discussion of [14], the spectrum of unthermalized decay products as a function of energy during IMD and RD epochs is given by

$$
\frac{d n}{d E} \approx \frac{3}{2}\left(\frac{H}{H_{R}}\right)^{\gamma} \frac{\rho_{S}\left(H_{R}\right)}{m_{S}} \frac{E^{1 / 2}}{m_{S}^{3 / 2}},
$$

where $\gamma=1$ during IMD ( $3 / 4$ during RD), $n(E)$ is the number density of decay products with energy less than $E$ and $H_{R}$ is the expansion rate at the onset of radiation domination. Thus $n(E) \propto E^{3 / 2}$ and so $80 \%$ of the decay products at a given time have energy between $E_{d}$ and $E_{d} / 3$, where $E_{d}$ is the energy of the most recent decay products. In addition, once thermalization by scattering begins, the lower energy decay products tend first to increase their energy by scattering from the more numerous higher-energy decay products in the spectrum $[15,16]$, so that they may be regarded as higherenergy decay products as far as thermalization is concerned. Thus we will consider the energy of the decay products at a given time to be approximately $E_{d}$. During IMD, $E_{d}$ $\approx m_{S} / 2$. Once the inflaton condensate has decayed away and the Universe enters the RD epoch, the energy of the dominant unthermalized decay products will be redshifted to $E_{d}$ $\approx\left(a_{R} / a\right) m_{S} / 2$, where $a_{R}$ is the scale factor at the onset of radiation domination [17].

The center of mass (CM) cross-section of the relativistic inflaton decay products is

$$
\sigma_{s c} \approx \frac{\alpha_{s c}^{2}}{E_{C M}^{2}},
$$

where $E_{C M} \approx 2 E_{d}$ and $\alpha_{s c}=g^{2} / 4 \pi$, where $g$ is a typical MSSM gauge or Yukawa coupling. Therefore the scattering rate of the inflaton decay products is $\Gamma_{s c}=n \sigma_{s c}$, where $n$ is the number density of inflaton decay products.

During IMD most of the decay products at a given scale factor are produced during the previous e-folding. The energy density in the inflaton condensate during IMD is

$$
\rho_{S}=\left(\frac{a_{e}}{a}\right)^{3} e^{-\Gamma_{d} t} \rho_{I},
$$

where $\Gamma_{d}$ is the decay rate of the inflaton, $a_{e}$ is the scale factor at the end of inflation and $\rho_{I}$ is the energy density during inflation, assumed constant. Therefore the number of inflatons which decay during an e-folding $\left(\delta t \approx H^{-1}\right.$ ) (and so the number of inflaton decay products produced) is

$$
n \approx \frac{\Gamma_{d}}{H} \frac{\rho_{S}}{m_{S}}
$$

for $\Gamma_{d} / H<1$. The condition for thermalization to occur during IMD, $\Gamma_{s c} \gtrsim H$, is then

$$
\frac{3 \alpha_{s c}^{2} M^{2} \Gamma_{d}}{m_{S}^{3}} \geqslant 1,
$$

where $M=M_{P l} / \sqrt{8 \pi}$ and we have used $E_{d}=m_{S} / 2$ and $\rho_{S}$ $\approx 3 M^{2} H^{2}$ during IMD. Equation (5) is independent of the scale factor, so if it is not satisfied immediately at the end of inflation, it will not be satisfied during IMD. Therefore for the case of perturbative inflaton decay, thermalization must either be instantaneous or must occur during RD.

After IMD, the number density and energy of the relativistic decay products is

$$
n=\left(\frac{a_{R}}{a}\right)^{3} n\left(a_{R}\right) ; \quad E_{d} \approx\left(\frac{a_{R}}{a}\right) \frac{m_{S}}{2} .
$$

The inflaton decay rate, $\Gamma_{d}$, may be expressed in terms of the reheating temperature, ${ }^{2} T_{R}$, as $\Gamma_{d}=k_{T_{R}} T_{R}^{2} / M_{P l}$, where $k_{T_{R}}=\left[4 \pi^{3} g\left(T_{R}\right) / 45\right]^{1 / 2}$ and $g\left(T_{R}\right)$ is the effective number of massless degrees of freedom in thermal equilibrium [20]. [In the following we will consider $k_{T_{R}} \approx 20$, corresponding to the field content of the MSSM with $g\left(T_{R}\right) \approx 200$.]

The thermalization condition $n \sigma_{s c} \geqslant H$ [where $H$ $=\left(a_{R} / a\right)^{2} H\left(a_{R}\right)$ during RD] then implies that thermalization occurs at scale factor $a_{t h}$ given by

\footnotetext{
${ }^{2}$ The reheating temperature is defined in the following to be the temperature of a thermalized Universe at the onset of radiation domination. It is therefore used generally to parametrize the energy density at the onset of radiation domination, even if the relativistic decay products at that time have not yet thermalized.
} 


$$
\frac{a_{t h}}{a_{e}} \approx \frac{m_{S}^{3}}{3 \alpha_{s c}^{2} M^{2} \Gamma_{d}}\left(\frac{H_{I}}{\Gamma_{d}}\right)^{2 / 3},
$$

with the corresponding temperature given by

$$
T_{t h} \approx \frac{3}{\sqrt{8 \pi}} \frac{k_{T_{R}} \alpha_{s c}^{2} M T_{R}^{3}}{m_{S}^{3}} .
$$

Here $H_{I}$ is the expansion rate during inflation.

\section{B. Scalar field squared expectation value of the inflaton decay product background}

When discussing the evolution of the RH sneutrino condensate, we will need the expectation value $\left\langle\Psi^{2}\right\rangle$, where $\Psi$ represents a generic real MSSM scalar in the inflaton decay product background [13].

(i) Unthermalized decay products during IMD. If we consider the average momentum of the scalar modes in the inflaton decay product background to be $\sim k$, then the average energy density of a real massless scalar field $\Psi$ is

$$
\left\langle\rho_{\Psi}\right\rangle=\left\langle\frac{1}{2} \Psi^{2}+\frac{1}{2}(\nabla \Psi)^{2}\right\rangle \approx k^{2}\left\langle\Psi^{2}\right\rangle .
$$

Thus with $k \approx E_{d} \approx m_{S} / 2$ and $\left\langle\rho_{\Psi}\right\rangle=f_{\Psi} \rho_{d}$, where $\rho_{d}$ $\approx\left(\Gamma_{d} / H\right) \rho_{S}$ is the energy density of the inflaton decay products during IMD and $f_{\Psi}$ is the fraction of the total inflaton decay product energy density in the real scalar field $\Psi$, we obtain

$$
\left\langle\Psi^{2}\right\rangle \approx \frac{f_{\Psi} \rho_{d}}{k^{2}} \approx \frac{12 f_{\Psi} \Gamma_{d} M^{2} H}{m_{S}^{2}}
$$

(ii) Unthermalized decay products during $R D$. In this case the energy of the dominant decay products redshifts as $E_{d}$ $\approx\left(a_{R} / a\right) m_{S} / 2$ while $\rho_{d} \propto a^{-4}$ for relativistic decay products, with $\rho_{d}\left(a_{R}\right) \approx \rho_{S}\left(a_{R}\right)$. Thus $\left\langle\Psi^{2}\right\rangle \propto \rho_{d} / E_{d}^{2} \propto a^{-2} \propto H$. Therefore the same relation, Eq. (10), between $\left\langle\Psi^{2}\right\rangle$ and $H$ also holds during RD.

(iii) Thermalized decay products. In this case the energy density of the inflaton decay products is $\rho_{d}$ $=\left[\pi^{2} g(T) T^{4} / 30\right]$, where for a real scalar field $g(T)=1$. Therefore, with $k \approx T$ for effectively massless thermalized particles, we find

$$
\left\langle\Psi^{2}\right\rangle \approx \gamma_{T} T^{2} ; \quad \gamma_{T}=\frac{\pi^{2}}{30}
$$

We note that in the case where the inflaton decay products thermalize immediately at the end of inflation, the temperature during IMD is related to $H$ by [20]

$$
T=k_{r}\left(M_{P l} H T_{R}^{2}\right)^{1 / 4} ; \quad k_{r}=\left(\frac{9}{5 \pi^{3} g(T)}\right)^{1 / 8} .
$$

\section{RIGHT-HANDED SNEUTRINO CONDENSATE EVOLUTION}

\section{A. Neutrino masses and the RH sneutrino scalar potential}

For simplicity we will consider a single neutrino generation. The superpotential of the RH neutrino superfield, $N$, is given by

$$
W_{\nu}=\lambda_{\nu} N H_{u} L+\frac{M_{N} N^{2}}{2},
$$

where $H_{u}$ and $L$ are the MSSM Higgs and charged lepton superfield $S U(2)_{L}$ doublets [2]. The corresponding scalar potential for the RH sneutrino is then $V(N)=\left(m_{\tilde{N}}^{2} / 2\right) N^{2}$ (with $N$ a conventionally normalized real scalar field), where $m_{\tilde{N}}^{2}=m_{o}^{2}+M_{N}^{2}+m_{e f f}^{2}$. Here $m_{o}^{2}$ is the conventional SUSY breaking mass squared term $\left(m_{o} \sim 100 \mathrm{GeV}\right)$ while $m_{\text {eff }}^{2}$ $\equiv \lambda_{\nu}^{2}\left\langle\Psi^{2}\right\rangle$ is the effective mass squared term due to the interaction of the RH sneutrinos with the inflaton decay product background [13]. In addition, we expect terms due to Planck-scale suppressed interactions. For now we will consider the evolution of the sneutrino condensate in the absence of such terms.

If $M_{N}=0$ we will have Dirac neutrino masses, with $m_{\nu}$ $=\lambda_{\nu} v_{u}$. The RH sneutrino mass is then given by $m_{\tilde{N}}^{2}=m_{o}^{2}$ $+m_{\text {eff }}^{2}$. If $M_{N} \gg \lambda_{\nu} v_{u}$ (where $v_{u}=\left\langle H_{u}\right\rangle$ ) we will have Majorana neutrino masses from the see-saw mechanism, $m_{\nu}$ $=\lambda_{\nu}^{2} v_{u}^{2} / M_{N}$, such that the Yukawa coupling $\lambda_{\nu}$ can be expressed as a function of $m_{\nu}$,

$$
\lambda_{\nu}=\left(\frac{m_{\nu} M_{N}}{v_{u}^{2}}\right)^{1 / 2} .
$$

The usual idea of the see-saw mechanism [1] is to consider the magnitude of $\lambda_{\nu}$ to be similar to the charged lepton Yukawa couplings, which requires e.g. $M_{N} \approx 10^{9} \mathrm{GeV}$ for $\lambda_{\nu} \approx \lambda_{\tau} \approx 10^{-2}$ and $m_{\nu} \approx 0.1 \mathrm{eV}$. However, other natural mass scales could also be of interest, for example $M_{N}$ $\sim 100 \mathrm{GeV}-1 \mathrm{TeV}$, as suggested by the electroweak scale and by the scale of the SUSY mass term $\mu H_{u} H_{d}$ of the MSSM superpotential [2].

\section{B. Conditions for RH sneutrino to act as a curvaton}

RH sneutrino oscillations begin once the RH sneutrino mass satisfies $m_{\tilde{N}} \gtrsim H$. We denote the scale factor at this time by $a_{o s c}$ and the homogeneous sneutrino expectation value by $N_{o s c}$. During inflation, in order to serve as a curvaton, the RH sneutrino must be effectively massless, $m_{\tilde{N}}$ $\ll H_{I}$. The quantum fluctuation of an effectively massless $\mathrm{RH}$ sneutrino mode at horizon crossing is given by $\delta N$ $\approx H_{I} / 2 \pi[20]$. In the case of a scalar potential consisting purely of a mass term $\left(\propto N^{2}\right)$, once the perturbation mode is stretched outside the horizon by the expansion of the Universe, its amplitude on subhorizon scales will evolve in the same way as the homogeneous field. This can be seen by 
considering $N=N_{o}+\delta N$, where $N_{o}$ is the homogeneous field and $\delta N$ is a perturbation of wave number $\mathbf{k}$. The equations of motion for these are

$$
\ddot{N}_{o}+3 H \dot{N}_{o}=-V^{\prime}\left(N_{o}\right)
$$

and

$$
\delta \ddot{N}+3 H \delta \dot{N}-\frac{\mathbf{k}^{2}}{a^{2}} \delta N=-V^{\prime \prime}\left(N_{o}\right) \delta N .
$$

For a mode outside the horizon, the $\mathbf{k}^{2} / a^{2}$ term $\left(\ll H^{2}\right)$ will effectively play no role in the evolution of the scalar field. Therefore, for $V(N) \propto N^{2}$, Eq. $\quad(15) \leftrightarrow$ Eq. (16) under $N_{o} \leftrightarrow \delta N$. Thus $N_{o}$ and $\delta N$ will evolve in the same way. Therefore $\delta N / N$ for a superhorizon perturbation will be fixed by its value at the onset of oscillations, $(\delta N / N)_{o s c}$. Once coherent oscillations of the RH sneutrino begin, the energy density in the RH sneutrino field will be proportional to its amplitude squared. Therefore if the energy density of the Universe becomes dominated by the RH sneutrino oscillations before the sneutrinos decay, the energy density perturbation when a given mode reenters the horizon will be given by

$$
\delta_{\rho} \equiv \frac{\delta \rho}{\rho} \approx\left(\frac{2 \delta N}{N}\right)_{o s c}=\frac{H_{I}}{\pi N_{I}} .
$$

(A more precise calculation gives the same result up to a factor of the order of 1 [9].) For $H_{I}$ and $N_{I}$ constant this corresponds to a scale-invariant perturbation spectrum. In order to account for the observed CMB temperature fluctuations, we then require that $\delta_{\rho} \approx 10^{-5}$ [22].

\section{Condensate evolution without Planck-suppressed terms}

The evolution of the RH sneutrino expectation value depends on the inflaton decay product background and reheating temperature. We will consider the case where the $\mathrm{RH}$ sneutrino begins coherent oscillations during the IMD epoch, $a_{\text {osc }}<a_{R}$. Oscillations begin at $H_{\text {osc }} \approx m_{\tilde{N}} \gtrsim 100 \mathrm{GeV}$. The condition $H_{R}<H_{\text {osc }} \approx m_{\tilde{N}}$ then implies an upper bound on $T_{R}$,

$$
T_{R} \lessgtr\left(\frac{m_{\tilde{N}} M_{P l}}{k_{T_{R}}}\right)^{1 / 2} \approx 7.8 \times 10^{9}\left(\frac{m_{\tilde{N}}}{100 \mathrm{GeV}}\right)^{1 / 2} \mathrm{GeV} .
$$

In particular, if $T_{R} \lesssim 10^{8} \mathrm{GeV}$, as would be required by the thermal gravitino upper bound $[21,24]$ if the inflaton decay products are thermalized at the onset of radiation domination, then RH sneutrino oscillations would generally begin during IMD. However, it is possible that thermalization of the relativistic decay products could occur at $T_{t h} \lesssim 10^{8}$ $\mathrm{GeV}$ even though $T_{R}>10^{8} \mathrm{GeV}$, in which case the thermal gravitino bound on $T_{R}$ could be evaded.

We will also assume that thermalization of the inflaton decay products occurs after the Universe becomes radiation dominated. This is true if Eq. (5) is not satisfied, which in turn requires that the inflaton mass satisfies

$$
m_{S} \gtrless 6 \times 10^{11} \alpha_{s c}^{2 / 3}\left(\frac{T_{R}}{10^{8} \mathrm{GeV}}\right)^{2 / 3} \mathrm{GeV},
$$

where $\alpha_{s c}$, being due to typical MSSM couplings, is not expected to be much smaller than 1 . We finally assume that $m_{\tilde{N}}^{2}$ is dominated by the time-independent terms when the sneutrino oscillations begin, $m_{\tilde{N}}^{2} \approx m_{\tilde{N}_{c}}^{2} \equiv m_{o}^{2}+M_{N}^{2}$. This requires that $m_{e f f}^{2}<m_{\tilde{N}_{c}}^{2}$ at $H_{o s c} \approx m_{\tilde{N}_{c}}$. Using $\left\langle\Psi^{2}\right\rangle$ for an unthermalized background during IMD, Eq. (10), this requires that

$$
m_{S}^{2} \gtrsim \frac{12 k_{T_{R}} f_{\Psi} \lambda_{\nu}^{2} M T_{R}^{2}}{\sqrt{8 \pi} m_{\tilde{N}_{c}}} .
$$

For the case of Dirac neutrinos this implies that

$$
\begin{aligned}
m_{S} \gtrsim & 1.1 \times 10^{5} f_{\Psi}^{1 / 2}\left(\frac{100 \mathrm{GeV}}{m_{\tilde{N}_{c}}}\right)^{1 / 2} \\
& \times\left(\frac{T_{R}}{10^{8} \mathrm{GeV}}\right)\left(\frac{m_{\nu}}{0.1 \mathrm{eV}}\right) \mathrm{GeV},
\end{aligned}
$$

where we have assumed $v_{u} \approx 100 \mathrm{GeV}$, while for the case of Majorana neutrino masses

$$
\begin{aligned}
m_{S} \gtrsim & \times 10^{11} f_{\Psi}^{1 / 2}\left(\frac{M_{N}}{m_{\tilde{N}_{c}}}\right)^{1 / 2} \\
& \times\left(\frac{T_{R}}{10^{8} \mathrm{GeV}}\right)\left(\frac{m_{\nu}}{0.1 \mathrm{eV}}\right)^{1 / 2} \mathrm{GeV}
\end{aligned}
$$

This assumption will give the largest RH sneutrino energy density at late times for a given $T_{R}$ and $N_{o s c}$, since the effect of having $m_{e f f}>m_{\tilde{N}_{c}}$ at $H \approx m_{\tilde{N}_{c}}$ would be to cause RH sneutrino oscillations to begin earlier and so to experience a greater dilution of the RH sneutrino energy density due to expansion. From now on we will consider $m_{\tilde{N}} \approx m_{\tilde{N}_{c}}$.

A fundamental condition for the RH sneutrino to play the role of a curvaton is that the RH sneutrinos decay after the Universe becomes dominated by the energy density in their coherent oscillations. The RH sneutrino decay rate is given by

$$
\Gamma_{\tilde{N} d} \approx \frac{\lambda_{\nu}^{2} m_{\tilde{N}}}{4 \pi}
$$

The RH sneutrinos decay once $\Gamma_{\tilde{N} d} \approx H$. At the onset of RH sneutrino oscillations, $\rho_{\tilde{N}} \approx m_{\tilde{N}}^{2} N_{o s c}^{2} / 2$ while $\rho_{S} \approx 3 H_{o s c}^{2} M^{2}$ $\approx 3 m_{\tilde{N}}^{2} M^{2}$. During IMD, the energy density in the inflaton oscillations and the RH sneutrino oscillations both evolve as 
$a^{-3}$. Thus $\rho_{\tilde{N}} / \rho_{S}$ is constant. Once the Universe is radiation dominated, the energy density in the dominant relativistic background, $\rho$, evolves as $a^{-4}$ while the energy density in the RH sneutrino evolves as $a^{-3}$. Therefore once $a>a_{R}$,

$$
\frac{\rho_{\tilde{N}}}{\rho}=\left(\frac{a}{a_{R}}\right)\left(\frac{\rho_{N}}{\rho_{S}}\right)_{o s c} \approx\left(\frac{a}{a_{R}}\right) \frac{N_{o s c}^{2}}{6 M^{2}} .
$$

Thus the Universe becomes RH sneutrino dominated once $H<H_{\text {dom }}$, where

$$
H_{d o m} \approx\left(\frac{N_{o s c}^{2}}{6 M^{2}}\right)^{2} H_{R} .
$$

The condition that RH sneutrino decay occurs after RH sneutrino domination, $\Gamma_{\tilde{N} d}<H_{d o m}$, then implies that

$$
m_{\tilde{N}} \lesssim\left(\frac{4 \pi k_{T_{R}} T_{R}^{2}}{\lambda_{\nu}^{2} M_{P l}}\right)\left(\frac{N_{o s c}^{2}}{6 M^{2}}\right)^{2} .
$$

We will refer to Eq. (26) as the late decay condition in the following.

For the case of Dirac neutrino masses the late decay condition becomes

$$
m_{\tilde{N}} \lessgtr\left(\frac{4 \pi v_{u}^{2}}{m_{\nu}^{2}}\right)\left(\frac{k_{T_{R}} T_{R}^{2}}{M_{P l}}\right)\left(\frac{N_{o s c}^{2}}{6 M^{2}}\right)^{2},
$$

which implies that

$$
\begin{aligned}
m_{\tilde{N}} \leqslant & 5.8 \times 10^{21}\left(\frac{0.1 \mathrm{eV}}{m_{\nu}}\right)^{2} \\
& \times\left(\frac{T_{R}}{10^{8} \mathrm{GeV}}\right)^{2}\left(\frac{N_{\text {osc }}}{M}\right)^{4} \mathrm{GeV} .
\end{aligned}
$$

Thus in order to have $m_{\tilde{N}} \geq m_{o} \approx 100 \mathrm{GeV}$ we require that $N_{\text {osc }} / M \gtrsim 1 \times 10^{-5}$. We will see that values of $N_{\text {osc }} / M$ in this range require some suppression of Planck-scale suppressed nonrenormalizable corrections to the $\mathrm{RH}$ neutrino superpotential.

For the case of Majorana neutrino masses the late decay condition becomes

$$
m_{\tilde{N}} \lessgtr\left(\frac{m_{\tilde{N}}}{M_{N}}\right)^{1 / 2}\left(\frac{4 \pi v_{u}^{2}}{m_{\nu}}\right)^{1 / 2}\left(\frac{k_{T_{R}} T_{R}^{2}}{M_{P l}}\right)^{1 / 2} \frac{N_{o s c}^{2}}{6 M^{2}},
$$

such that

$$
\begin{aligned}
m_{\tilde{N}} \leqslant & 7.6 \times 10^{5}\left(\frac{m_{\tilde{N}}}{M_{N}}\right)^{1 / 2}\left(\frac{0.1 \mathrm{eV}}{m_{\nu}}\right)^{1 / 2} \\
& \times\left(\frac{T_{R}}{10^{8} \mathrm{GeV}}\right)\left(\frac{N_{o s c}}{M}\right)^{2} \mathrm{GeV} .
\end{aligned}
$$

We will restrict attention to $\mathrm{RH}$ neutrino masses $M_{N} \geq m_{o}$ $\approx 100 \mathrm{GeV}$, since masses smaller than this would have little motivation from neutrino mass models or natural particle physics scales. In this case $m_{\tilde{N}} \approx M_{N}$. Equation (30) then shows that a right-handed Majorana sneutrino curvaton with $M_{N} \gtrsim 100 \mathrm{GeV}$ is possible only if $N_{\text {osc }}$ is not very small compared with the reduced Planck scale $M$. $\left(N_{o s c} / M\right.$ $\geq 0.01$ if $T_{R} \lesssim 10^{8} \mathrm{GeV}$.) It also shows that $T_{R}$ cannot be very small compared with the thermal gravitino upper bound of $10^{8} \mathrm{GeV}$ if $N_{\text {osc }} \leq M$. The requirement that $N_{\text {osc }}$ is not very small compared with $M$ is a strong constraint on a Majorana RH sneutrino curvaton, since, as we discuss below, it requires a high degree of suppression of Planck-scale suppressed contributions to the RH sneutrino superpotential.

From Eq. (17) and the density perturbation constraint, $\delta_{\rho} \approx 10^{-5}$, the expansion rate during inflation is $H_{I}$ $=\pi \delta_{\rho} N_{I} \geqslant 10^{9} \mathrm{GeV}$ if $N_{I} \approx N_{\text {osc }} \gtrsim 1 \times 10^{-5} \mathrm{M}$, corresponding to the case of Dirac neutrino masses. This can be substantially lower than the typical value of the expansion rate during inflation found in conventional SUSY inflation models, $H_{I} \approx 10^{13} \mathrm{GeV}$ [25-27]. For the case of Majorana neutrino masses with $N_{I} \approx N_{\text {osc }} \geqslant 0.01 \mathrm{M}$, the corresponding bound on $H_{I}$ is $H_{I} \gtrsim 7 \times 10^{11} \mathrm{GeV}$. These bounds assume that $N$ does not evolve significantly from the end of inflation to the onset of sneutrino oscillations, so that $N_{\text {osc }} \approx N_{I}$. We will see that the range of allowed $H_{I}$ can be increased if this assumption is altered.

\section{Effect of Planck-suppressed terms}

In general, in addition to the globally SUSY scalar potential we expect (in the absence of specific symmetries) terms suppressed by powers of the reduced Planck mass, $M$ $=M_{P l} / \sqrt{8 \pi}$, corresponding to the natural scale of supergravity (SUGRA) corrections $[3,19]$. Thus we expect Planckscale suppressed non-renormalizable terms to appear in the RH neutrino superpotential. We also expect contributions to the mass squared term of the form $c H^{2}$, where $|c|$ is modeldependent but expected to be of the order of 1 in the simplest models. These arise from terms in the full Lagrangian of the form $\left(1 / M^{2}\right) \int d^{4} \theta S^{\dagger} S N^{\dagger} N=\left|F_{S}\right|^{2} / M^{2}$, where $S$ is the inflaton field (or any other field with a non-zero F-term contributing to the energy density of the Universe).

\section{1. $\mathrm{cH}^{2}$ corrections}

During inflation the value of $|c|$ is constrained by the deviation of the curvaton perturbation from scale-invariance. We consider the RH sneutrino potential during inflation to be

$$
V(N) \approx \frac{1}{2} c H^{2} N^{2} .
$$

The expansion rate during inflation is then

$$
H_{I}^{2}=\frac{1}{3 M^{2}}\left(\rho_{S}+\frac{c H_{I}^{2} N^{2}}{2}\right)
$$


where $\rho_{S}$ is the energy density of the inflaton field, which we assume to be constant.

The index $n$ of the perturbation spectrum as a function of the present wave number $k$ is given by

$$
n=1+\frac{2 k}{\delta_{\rho}} \frac{d \delta_{\rho}}{d k},
$$

such that $\delta \rho / \rho \propto k^{(n-1) / 2}$ and $n=1$ corresponds to scaleinvariance. $\delta_{\rho}$ will remain constant once the perturbation is outside the horizon, since both $N$ and $\delta N$ evolve in the same way for $V(N) \propto N^{2}$. Therefore we have

$$
\delta_{\rho} \equiv \frac{2 \delta N}{N}=\left(\frac{H_{I}}{\pi N}\right)_{a_{\lambda}},
$$

where $a_{\lambda}$ is the scale-factor at which a perturbation of wavelength $\lambda$ exits the horizon.

The value of $N$ as a function of the scale factor during inflation is given by the solution of

$$
\ddot{N}+3 H \dot{N}=-c H^{2} N
$$

With $H \propto a^{-m}$, this has a solution of the form $N \propto a^{\gamma}$, where

$$
\gamma=\frac{1}{2}\left[-(3-m)+\sqrt{(3-m)^{2}-4 c}\right] .
$$

Thus during inflation $(m=0)$ the solution corresponds to

$$
\gamma=\frac{1}{2}[-3+\sqrt{9-4 c}]
$$

If $|4 c| \ll 9$ then during inflation $\gamma \approx-c / 3$ and so $N / N_{\lambda}$ $=\left(a / a_{\lambda}\right)^{-c / 3}$, where $N_{\lambda}$ is the value at $a_{\lambda}$. Thus for a given $N$ and $a$ (for example, their values at the end of inflation), $N_{\lambda} \propto a_{\lambda}^{-c / 3}$. The wave number at present is related to the scale factor at the horizon exit $\left(\lambda \approx H_{I}^{-1}\right)$ by $k$ $=2 \pi H_{I} a_{\lambda} / a_{p}$, where $a_{p}$ is the scale factor at present. Thus

$$
\frac{d N_{\lambda}}{d k}=\frac{d N_{\lambda}}{d a_{\lambda}} \frac{d a_{\lambda}}{d k}=-\frac{c N_{\lambda}}{3 k} .
$$

For the case where the energy density is dominated by $\rho_{S}$, we have

$$
\frac{d \delta_{\rho}}{d N_{\lambda}} \approx-\frac{H}{\pi N_{\lambda}^{2}} .
$$

Therefore

$$
n=1+\frac{2 k}{\delta_{\rho}} \frac{d \delta_{\rho}}{d N_{\lambda}} \frac{d N_{\lambda}}{d k} \approx 1+\frac{2 c}{3} .
$$

Note that $c>0(<0)$ results in a blue (red) spectrum of perturbations. Observation requires that $|\Delta n|<0.1$ [22]. Therefore during inflation we must have

$$
|c|<0.15\left|\frac{\Delta n}{0.1}\right| .
$$

If $|c|$ is close to this upper bound then a significant blue or red perturbation spectrum is expected. We note that this would allow the RH sneutrino curvaton scenario to be consistent with the recent observation by WMAP of a blue perturbation spectrum on comoving scales of the order of 500 Mpc, with $n=1.10_{-0.06}^{+0.07}[23]$.

Thus $|c|$ is constrained to be not much larger than 0.1 during inflation. This is significantly larger than the value $|c| \sim 1$ expected on dimensional grounds in SUGRA models [18]. This problem is similar to the conventional " $\eta$-problem" encountered in SUSY inflation models [2527], where order $H^{2}$ corrections lead both to a large deviation from scale-invariance and to insufficient slow-roll inflation [18]. It has a natural solution in the case of SUSY D-term hybrid inflation (driven by the energy density of a Fayet-Illiopoulos D-term) [25], in which case $|F|=0$ and so $|c|=0$ during inflation [although a nonzero value is expected once inflation ends and coherent inflaton oscillations begin, since $\int d^{4} \theta S^{\dagger} S \Phi^{\dagger} \Phi=\left(\left|\partial_{\mu} S\right|^{2}+\left|F_{S}\right|^{2}+\ldots\right)|\Phi|^{2}$, with $\Phi$ a general scalar superfield]. Since in the RH sneutrino curvaton scenario inflation must still be driven by an inflaton, it is possible that the mechanism which suppresses $|c|$ for the inflaton during inflation also suppresses $|c|$ for the curvaton.

After inflation we generally expect a nonzero F-term from the energy density of the coherently oscillating inflaton field. The solution of Eq. (35) during IMD corresponds to Eq. (36) with $m=3 / 2$. Thus assuming that $N$ has a constant value during inflation, $N_{I}$, we find

$$
\frac{N_{o s c}}{N_{I}}=\left(\frac{a_{o s c}}{a_{e}}\right)^{\gamma}=\left(\frac{H_{I}}{H_{o s c}}\right)^{2 \gamma / 3},
$$

where $H_{o s c} \approx m_{\tilde{N}}$.

We previously derived lower bounds on the value of $H_{I}$ compatible with the observed density perturbations based on the assumption that $N_{o s c} \approx N_{I}$. However, if $c<0$ during IMD then the growth of $N$ after inflation will allow a wider range of $H_{I}$ to be compatible with a given value of $N_{\text {osc }}$,

$$
\frac{2 \gamma}{3}=\frac{\ln \left(\frac{\pi \delta_{\rho} N_{o s c}}{H_{I}}\right)}{\ln \left(\frac{H_{I}}{m_{\tilde{N}}}\right)}
$$

where we have used $N_{I} \approx H_{I} / \pi \delta_{\rho}$ in Eq. (42). For example, for the case of Dirac neutrino masses, with $\delta_{\rho} \approx 10^{-5}, m_{\tilde{N}}$ $\approx 100 \mathrm{GeV}$ and $N_{o s c} \approx 10^{-4} M$, it is possible to have $H_{I}$ $\approx 10^{6} \mathrm{GeV}$ if $\gamma=1.45$, corresponding to $c=-4.3$. This also shows that $|c|$ need not be small compared with 1 after inflation.

We conclude that the $c H^{2}$ correction to the RH sneutrino mass squared term is no more problematical for the curvaton than for the conventional inflaton: we require that $|c| \lesssim 0.1$ during inflation, in which case a significantly blue or red 
perturbation spectrum can arise depending on the sign and magnitude of $c$. After inflation $|c|$ need not be small compared with 1 (in the case where $c<0$ ) and the $c H^{2}$ term can even widen the range of expansion rate and energy density during inflation which is compatible with the observed density perturbations.

\section{Non-renormalizable superpotential corrections}

We next consider adding Planck scale-suppressed nonrenormalizable terms (NRTs) to the RH sneutrino superpotential,

$$
W_{N}=\frac{1}{2} M_{N} N^{2}+\frac{\lambda_{n} N^{n}}{n ! M^{n-3}},
$$

where $n$ ! is a symmetry factor and $\lambda_{n} \approx 1$. For large enough $|N|$ the scalar potential becomes dominated by the NRT contribution,

$$
V(N) \approx \frac{\lambda_{n}^{2} N^{2(n-1)}}{2^{n-1}(n-1) !^{2} M^{2(n-3)}} .
$$

The effect of the NRTs is to place an upper limit on the value of $N_{o s c}$. During inflation, the effective mass $V^{\prime \prime}(N)$ must be small compared with $H^{2}$. After inflation and during IMD, $H^{2} \propto a^{-3}$. Therefore $V^{\prime \prime}(N)$ may become larger than $H^{2}$, at which point the $N$ field will begin to evolve. $N$ will then track the value at which $V^{\prime \prime}(N) \approx H^{2}$, since as $H^{2}$ decreases below $V^{\prime \prime}(N)$, the rate of roll of $N$ will increase until the rate of decrease of $V^{\prime \prime}(N)$ matches the rate of the decrease of $H^{2}$. In particular, when $N$ will first begin to slow-roll we have $3 H \dot{N} \approx-V^{\prime}(N)$. For $V(N) \propto N^{2(n-1)}$, this has a solution $N$ $\propto a^{3 /(4-2 n)}$, such that $V^{\prime \prime}(N) \propto a^{-3} \propto H^{2}$. So the slow-rolling solution will be such that $V^{\prime \prime}(N)$ tracks $H^{2}$ and is of the same order of magnitude as $H^{2}$. Thus the value of $N$ at which $V^{\prime \prime}(N) \approx H^{2}$ places an upper limit on $N$ for a given value of $H, N_{\text {lim }}$, given by

$$
\frac{N_{l i m}}{M} \approx \frac{\alpha_{n}}{\lambda_{n}^{1 /(n-2)}}\left(\frac{H}{M}\right)^{1 /(n-2)},
$$

where $\alpha_{n}$ is a constant of order 1 . Therefore for $N_{\text {lim }}$ to be greater than $N_{\text {osc }}$ at $H_{\text {osc }}$ we require that $n$ is greater than $n_{\text {lim }}$, where

$$
n_{\text {lim }}=2+\frac{\ln \left(\frac{H_{\text {osc }}}{M}\right)}{\ln \left(\frac{\lambda_{n}^{1 /(n-2)} N_{\text {osc }}}{\alpha_{n} M}\right)} .
$$

For the case of Dirac neutrino masses, we require from the late decay condition that $N_{\text {osc }} / M \gtrsim 1 \times 10^{-5}$. Thus with $H_{o s c} \approx m_{\tilde{N}}=100 \mathrm{GeV}, N_{o s c} / M \approx 1 \times 10^{-5}$ and $\lambda_{n} \approx \alpha_{n} \approx 1$, we require that $n>n_{\text {lim }}=5.3$. Thus a suppression of Planckscale suppressed NRTs in the RH neutrino superpotential of dimension less than 6 is required in this case. This might be achieved by a modest discrete symmetry.

For the case of Majorana neutrino masses, with $H_{\text {osc }}$ $\approx m_{\tilde{N}}=100 \mathrm{GeV}, N_{o s c} / M \approx 0.01$ and $\lambda_{n} \approx \alpha_{n} \approx 1$, we require that $n>n_{\text {lim }}=10.2$. This is a significant problem for a Majorana RH sneutrino curvaton. It requires a high degree of suppression of Planck-suppressed nonrenormalizable terms, eliminating all NRTs in the RH neutrino superpotential up to $n<11$. However, as we will discuss, there is likely to be a more severe problem for the Majorana RH sneutrino curvaton, namely the survival of the RH sneutrino condensate in the inflaton decay product background.

Although Planck scale-suppressed NRTs appear to disfavor a RH sneutrino curvaton, it should be noted that conventional SUSY inflation models also have problems with Planck scale-suppressed NRTs. Chaotic inflation models require that the inflaton expectation value is greater than $M$ [27], while SUSY hybrid inflation models require that $N$ is close to $M$ when scales corresponding to observed cosmic microwave background (CMB) perturbations exit the horizon (assuming natural values of the renormalizable gauge and Yukawa couplings) [25,26]. In this sense the RH sneutrino curvaton may be no more problematical than conventional SUSY inflation models with respect to Planck scale-suppressed superpotential terms.

\section{E. Decay temperature of the Dirac and Majorana RH sneutrino condensate}

The neutrino mass is related to the Yukawa coupling by $m_{\nu}=\lambda_{\nu} v_{u}$. The RH sneutrino mass is simply given by the SUSY breaking mass term, $m_{\tilde{N}}=m_{o} \approx 100 \mathrm{GeV}$. Thus the temperature of the Universe when the condensate decays, $T_{\tilde{N} d}$, is given by $\Gamma_{\tilde{N} d} \approx H\left(T_{\tilde{N} d}\right)$, where $H(T)=k_{T} T^{2} / M_{P l}$ and $k_{T}=\left[4 \pi^{3} g(T) / 45\right]^{1 / 2}$. Thus with the RH sneutrino decay rate given by Eq. (23), the temperature of RH sneutrino decay is, in general,

$$
T_{\tilde{N} d}=\left(\frac{\lambda_{\nu}^{2} m_{\tilde{N}} M_{P l}}{4 \pi k_{T_{\tilde{N} d}}}\right)^{1 / 2} .
$$

For the case of the Dirac RH sneutrinos, Eq. (48) implies that

$$
\begin{aligned}
T_{\tilde{N} d} & =\left(\frac{m_{\nu}^{2} m_{\tilde{N}} M_{P l}}{4 \pi v_{u}^{2} k_{T_{\tilde{N} d}}}\right)^{1 / 2} \\
& =4.4\left(\frac{m_{\nu}}{0.1 \mathrm{eV}}\right)\left(\frac{m_{\tilde{N}}}{100 \mathrm{GeV}}\right)^{1 / 2} \mathrm{MeV}
\end{aligned}
$$

where we have used $v_{u} \approx 100 \mathrm{GeV}$ and $k_{T_{\tilde{N} d}} \approx 5$, corresponding to $\gamma, e^{ \pm}$and $\nu_{i}(i=1,2,3)$ as light degrees of freedom [20]. Thus the Dirac RH sneutrino condensate typically decays in the temperature range $1-10 \mathrm{MeV}$. (Note that if the energy density of the RH sneutrino condensate dominates the energy density of the Universe when it decays, $T_{\tilde{N} d}$ should then be interpreted as the temperature to which the Universe reheats after the condensate decays.) Since the Dirac RH sneutrino condensate decays well below the temperature at 
which weakly interacting particles of mass of the order of $m_{W}$ freeze out of chemical equilibrium $\left(T_{\text {freeze }} \approx 1\right.$ $-10 \mathrm{GeV}$ ), an important constraint on the Dirac RH sneutrino curvaton is the requirement that the lightest supersymmetric particles (LSPs) produced in decay of the RH sneutrino condensate do not overclose the Universe. This requires that the LSPs decay before nucleosynthesis at $T$ $\approx 1 \mathrm{MeV}$, in order that the light element abundances are not disrupted by photo-dissociation due to LSP decay cascades [24]. Therefore the LSP lifetime must satisfy

$$
\tau_{L S P} \lesssim H^{-1}(T \approx 1 \mathrm{MeV}) \approx 1 \mathrm{~s} .
$$

Thus although the LSP must be unstable its lifetime can be much longer than the time required to escape particle detectors $\left(\sim 10^{-8} \mathrm{~s}\right)$, in which case experimental searches for SUSY particles would be unaffected. A wider range of LSP candidates would be allowed than in the $R$-parity conserving case where the LSP properties are constrained by the thermal relic cold dark matter density. This might be testable at future colliders such as the CERN Large Hadron Collider.

For the case of Majorana RH sneutrinos, Eq. (48) implies that

$$
\begin{aligned}
T_{\tilde{N} d} & =\left(\frac{m_{\nu} m_{\tilde{N}}^{2} M_{P l}}{4 \pi v_{u}^{2} k_{T_{\tilde{N}}}}\right)^{1 / 2} \\
& =2.2 \times 10^{3}\left(\frac{m_{\nu}}{0.1 \mathrm{eV}}\right)^{1 / 2}\left(\frac{m_{\tilde{N}}}{100 \mathrm{GeV}}\right) \mathrm{GeV} .
\end{aligned}
$$

Thus since $m_{\tilde{N}}$ typically will not be much larger than $1 \mathrm{TeV}$ as a result of the late decay condition, Eq. (30), we expect that $T_{\tilde{N} d} \sim 10^{4} \mathrm{GeV}$ for the Majorana RH sneutrino condensate.

\section{F. Thermalization of the right-handed sneutrino condensate}

We have been assuming throughout the preceding discussion that the RH sneutrino condensate survives in the environment of the inflaton decay products. However, it is possible that RH sneutrinos in the condensate could be inelastically scattered by collisions with the MSSM particles in the inflaton decay product background. If the relativistic particles in the background have energy $E_{d}$, then the scattering cross-section of a RH sneutrino, at rest in the condensate, from a relativistic particle in the inflaton decay product background via Higgs(ino) or (s)lepton exchange is expected to be

$$
\sigma_{\tilde{N} s c} \approx \frac{\alpha_{\nu} \alpha_{g}}{E_{C M}^{2}},
$$

where $\alpha_{\nu}=\lambda_{\nu}^{2} / 4 \pi, \alpha_{g}=g^{2} / 4 \pi$, where $g$ is a typical MSSM gauge/Yukawa coupling and $E_{C M} \approx \sqrt{E_{d} m_{\tilde{N}}}$ is the center of mass energy of the process. The scattering rate from the decay product background is then $\Gamma_{\tilde{N} s c}=n \sigma_{\tilde{N} s c}$, where $n$ is the number density of particles in the inflaton decay product background. The largest scattering rate will occur for the largest $n$ and smallest $E_{d}$, which corresponds to thermalized decay products $\left(T_{\sim}^{<} T_{t h}\right)$. In this case $n=g(T) T^{3} / \pi^{2}$ and $E_{d} \approx T$ [20]. Therefore

$$
\Gamma_{\tilde{N} s c} \approx \frac{g(T) \alpha_{\nu} \alpha_{g}}{\pi^{2}} \frac{T^{2}}{m_{\tilde{N}}}
$$

Then assuming that thermalization of the inflaton decay products occurs during RD, the condition that the condensate is unthermalized is $\Gamma_{\tilde{N} s c} \lesssim H(T) \equiv k_{T} T^{2} / M_{P l}$, which implies

$$
\lambda_{\nu} \lesssim\left(\frac{4 \pi^{3} k_{T}}{\alpha_{g} g(T)}\right)^{1 / 2}\left(\frac{m_{\tilde{N}}}{M_{P l}}\right)^{1 / 2} .
$$

For the case of Dirac neutrino masses, this condition becomes

$$
\begin{aligned}
m_{\nu} & \lessgtr\left(\frac{4 \pi^{3} k_{T} v_{u}^{2}}{\alpha_{g} g(T)}\right)^{1 / 2}\left(\frac{m_{\tilde{N}}}{M_{P l}}\right)^{1 / 2} \\
& \approx \frac{1.0}{\alpha_{g}^{1 / 2}}\left(\frac{m_{\tilde{N}}}{100 \mathrm{GeV}}\right)^{1 / 2}\left(\frac{v_{u}}{100 \mathrm{GeV}}\right) \mathrm{keV},
\end{aligned}
$$

where we have used $g(T) \approx 200$ and $k_{T} \approx 20$. Therefore, since $m_{\nu} \approx 0.1 \mathrm{eV}$, the Dirac RH sneutrino condensate will not be thermalized.

For the case of Majorana neutrino masses, the nonthermalization condition becomes

$$
m_{\nu} \lessgtr\left(\frac{4 \pi^{3} k_{T}}{\alpha_{g} g(T)}\right) \frac{v_{u}^{2}}{M_{P l}} \approx \frac{10^{-5}}{\alpha_{g}}\left(\frac{v_{u}}{100 \mathrm{GeV}}\right)^{2} \mathrm{eV} .
$$

Thus typically we expect that this will not be satisfied. For example, if we consider $\alpha_{g}$ to correspond to the top quark Yukawa (RH sneutrinos scattering from thermal top quarks via $H_{u}$ exchange) then $\alpha_{g} \approx 0.1$ and so $m_{\nu} \lesssim 10^{-4} \mathrm{eV}$ would be required to evade thermalization. Therefore with $m_{\nu}$ $\approx 0.1 \mathrm{eV}$ the Majorana RH sneutrino condensate will be thermalized at $T_{t h}$, when the inflaton decay product background thermalizes.

One possible escape from this conclusion is that the inflaton decay product background could remain unthermalized until the energy density of the sneutrino condensate comes to dominate the energy density of the Universe. Since the Majorana RH sneutrino condensate decays at a temperature typically around $10^{4} \mathrm{GeV}$, if $T_{t h} \lesssim 10^{4} \mathrm{GeV}$ then the Majorana RH sneutrino curvaton may remain a possibility. For the case of perturbative inflaton decays, the thermalization temperature of the inflaton decay product background $T_{t h}$, Eq. (8), is proportional to $\left(T_{R} / m_{S}\right)^{3}$. Therefore a very low background thermalization temperature is a possibility if $T_{R} \ll m_{S}$.

The above applies to condensate thermalization during $\mathrm{RD}$. It is straightforward to see that if condensate thermalization does not occur during RD then it will not occur earlier during IMD. If we assume that the background is thermalized during IMD (which should give the largest rate of condensate thermalization), then since during IMD $\Gamma_{\tilde{N} s c} \propto T^{2}$ and 
$H \propto T^{4}$, it follows that once $T>T_{R}$ the thermalization condition $\Gamma_{\tilde{N} s c} \gtrsim H$ will become more difficult to satisfy. Thus if the RH sneutrino condensate does not thermalize during RD it will not thermalize at all.

In discussing the thermalization rate, we have implicitly assumed that the mass of the $H_{u}$ and $L$ fields coupling directly to the RH sneutrino condensate is small compared with $T$ and $E_{C M} \approx\left(T m_{\tilde{N}}\right)^{1 / 2}$. The effective mass is given by $\lambda_{\nu}\langle N\rangle$, where $\langle N\rangle$ is the amplitude of the coherent oscillations. In general $\langle N\rangle$ is given by

$$
\langle N\rangle=\left(\frac{a_{o s c}}{a}\right)^{3 / 2} N_{o s c} \approx\left(\frac{T}{T_{R}}\right)^{3 / 2}\left(\frac{H_{R}}{H_{o s c}}\right) N_{o s c} .
$$

Thus for the case of Majorana RH sneutrinos the effective mass is given by

$$
\lambda_{\nu}\langle N\rangle=\frac{k_{T} T^{3 / 2} T_{R}^{1 / 2}}{\sqrt{8 \pi}}\left(\frac{m_{\nu}}{m_{\tilde{N}} v_{u}^{2}}\right)^{1 / 2}\left(\frac{N_{o s c}}{M}\right),
$$

which implies that

$$
\begin{aligned}
\lambda_{\nu}\langle N\rangle= & 4 \times 10^{2}\left(\frac{m_{\nu}}{0.1 \mathrm{eV}}\right)^{1 / 2}\left(\frac{T}{10^{4} \mathrm{GeV}}\right)^{3 / 2} \\
& \times\left(\frac{T_{R}}{10^{8} \mathrm{GeV}}\right)^{1 / 2}\left(\frac{100 \mathrm{GeV}}{m_{\tilde{N}}}\right)^{1 / 2}\left(\frac{N_{\text {osc }}}{M}\right) \mathrm{GeV},
\end{aligned}
$$

where we have used $k_{T} \approx 20$ and $v_{u}=100 \mathrm{GeV}$. Thus for $N_{\text {osc }} / M<1, m_{\tilde{N}} \gtrsim 100 \mathrm{GeV}$ and for a decay temperature of $10^{4} \mathrm{GeV}$ for the Majorana RH sneutrino, we find that $\lambda_{\nu}\langle N\rangle$ is smaller than $T$ and $E_{C M}$ and so may be neglected when considering condensate thermalization.

For the case of Dirac RH sneutrinos the effective mass is given by

$$
\lambda_{\nu}\langle N\rangle=\frac{k_{T} T^{3 / 2} T_{R}^{1 / 2} m_{\nu}}{\sqrt{8 \pi} v_{u} m_{\tilde{N}}}\left(\frac{N_{o s c}}{M}\right),
$$

which implies that

$$
\begin{aligned}
\lambda_{\nu}\langle N\rangle= & 3 \times 10^{-15}\left(\frac{m_{\nu}}{0.1 \mathrm{eV}}\right)\left(\frac{T}{10 \mathrm{MeV}}\right)^{3 / 2} \\
& \times\left(\frac{T_{R}}{10^{8} \mathrm{GeV}}\right)^{1 / 2}\left(\frac{100 \mathrm{GeV}}{m_{\tilde{N}}}\right)\left(\frac{N_{\text {osc }}}{M}\right) \mathrm{GeV} .
\end{aligned}
$$

Thus the effective mass is generally negligible in the case of Dirac RH sneutrinos.

\section{CONCLUSIONS}

We have considered the possibility that a RH sneutrino could play the role of a curvaton in the cosmology of the MSSM extended to accommodate neutrino masses.
In the case of a Dirac RH sneutrino, the expectation value of the RH sneutrino at the onset of its coherent oscillations must satisfy $N_{o s c} / M \gtrsim 10^{-5}$, in order that the energy density of the RH sneutrino condensate dominates the Universe when it decays. As a result, Planck-scale corrections to the RH neutrino superpotential must be suppressed, eliminating all superpotential terms $\propto N^{n}$ with $n<6$. $c H^{2}$ corrections to the RH sneutrino mass squared must also be suppressed during inflation $(|c| \lesssim 0.1)$. The inflaton sector of the Dirac RH sneutrino curvaton scenario can have a much smaller expansion rate during inflation than conventional SUSY inflation models, with $H_{I} \approx 10^{9} \mathrm{GeV}$ possible. (Including the effect of a negative $c H^{2}$ term after inflation, values of $H_{I}$ as small as $10^{6} \mathrm{GeV}$ or less are possible.)

In addition, depending on the sign and magnitude of the $c H^{2}$ correction, it is possible to have a significantly blue ( $c$ $>0)$ or red $(c<0)$ perturbation spectrum. The recent suggestion from WMAP observations [23] of a blue perturbation spectrum on comoving scales of the order of $500 \mathrm{Mpc}$ could therefore be accommodated within the curvaton scenario.

The late decay of the Dirac RH sneutrino condensate (at $T \approx 1-10 \mathrm{MeV}$ ) requires that $R$ parity be broken and that the LSP decays before nucleosynthesis, corresponding to a lifetime shorter than $1 \mathrm{~s}$. (Since this would result in the loss of LSP cold dark matter, a new dark matter candidate would also be needed.) Thus the Dirac curvaton scenario predicts that LSP properties will typically be inconsistent with thermal relic cold dark matter.

In the case of a Majorana RH sneutrino curvaton, we find that the requirement that the RH sneutrino condensate dominates the energy density of the Universe before it decays implies that $N_{o s c} / M \geqslant 0.01$. This imposes a strong constraint on Planck-scale suppressed contributions to the RH neutrino superpotential, requiring elimination of all terms $\propto N^{n}$ with $n<11$. However, a more severe problem may arise from scattering of the condensate sneutrinos by particles in the thermal background, since it is likely that the Majorana RH sneutrino condensate will be thermalized as soon as the inflaton decay product background thermalizes. Thus if the inflaton decay products thermalize earlier than the time of RH sneutrino condensate domination of the energy density then the Majorana curvaton scenario will be ruled out. Only a sufficiently low background thermalization temperature could evade this conclusion. Therefore, although not absolutely excluded, the Majorana RH sneutrino curvaton scenario appears to be more difficult to implement than the Dirac curvaton scenario.

In conclusion, we find that a RH sneutrino can serve as the source of the density perturbations leading to structure formation. For the favored case of a Dirac RH sneutrino, this requires some suppression of its Planck-scale suppressed superpotential self-interactions together with sufficiently rapid $R$-parity violation. If these conditions are met, the Dirac $\mathrm{RH}$ sneutrino would provide us with the only curvaton candidate which is strongly motivated by particle physics.

Note added. After completing this work we became aware of [28] and [29], which also discuss the case of a Majorana RH sneutrino curvaton. 
[1] For a recent review, see R.N. Mohapatra, hep-ph/0211252.

[2] H.P. Nilles, Phys. Rep. 110, 1 (1984).

[3] M. Dine, L. Randall, and S. Thomas, Nucl. Phys. B458, 291 (1996).

[4] K. Enqvist and A. Mazumdar, hep-ph/0209244.

[5] I.A. Affleck and M. Dine, Nucl. Phys. B249, 361 (1985).

[6] S. Mollerach, Phys. Rev. D 42, 313 (1990).

[7] K. Enqvist and M.S. Sloth, Nucl. Phys. B626, 395 (2002).

[8] T. Moroi and T. Takahashi, Phys. Lett. B 522, 215 (2001); Phys. Rev. D 66, 063501 (2002).

[9] D.H. Lyth and D. Wands, Phys. Lett. B 524, 5 (2002); D.H. Lyth, C. Ungarelli, and D. Wands, Phys. Rev. D 67, 023503 (2003).

[10] N. Bartolo and A.R. Liddle, Phys. Rev. D 65, 121301(R) (2002).

[11] K. Enqvist, S. Kasuya, and A. Mazumdar, Phys. Rev. Lett. 90, 091302 (2003).

[12] M. Bastero-Gil, V. Di Clemente, and S.F. King, Phys. Rev. D 67, 103516 (2003).

[13] R. Allahverdi, B.A. Campbell, and J.R. Ellis, Nucl. Phys. B579, 355 (2000).

[14] J. McDonald, Phys. Rev. D 61, 083513 (2000).

[15] S. Davidson and S. Sarkar, J. High Energy Phys. 11, 012 (2000).

[16] R. Allahverdi, Phys. Rev. D 62, 063509 (2000); R. Allahverdi and M. Drees, ibid. 66, 063513 (2002).

[17] S. Davidson and A. Ibarra, Phys. Lett. B 535, 35 (2002).
[18] M. Dine, W. Fischler, and D. Nemeschansky, Phys. Lett. 136B, 169 (1984); G.D. Coughlan, R. Holman, P. Ramond, and G.G. Ross, ibid. 140B, 44 (1984); O. Bertolami and G.G. Ross, Phys. Lett. B 183, 163 (1987); M. Dine, L. Randall, and S. Thomas, Phys. Rev. Lett. 75, 398 (1995); G. Dvali, Phys. Lett. B 355, 78 (1995).

[19] E.J. Copeland, A.R. Liddle, D.H. Lyth, E.D. Stewart, and D. Wands, Phys. Rev. D 49, 6410 (1994).

[20] E.W. Kolb and M.S. Turner, The Early Universe (AddisonWesley, Reading, MA, 1990).

[21] J. Ellis, J.E. Kim, and D.V. Nanopoulos, Phys. Lett. 145B, 181 (1984).

[22] M. Tegmark, M. Zaldarriaga, and A.J.S. Hamilton, Phys. Rev. D 63, 043007 (2001).

[23] H.V. Peiris et al., astro-ph/0302225.

[24] S. Sarkar, Rep. Prog. Phys. 59, 1493 (1996).

[25] E. Halyo, Phys. Lett. B 387, 43 (1996); P. Binetruy and G. Dvali, ibid. 388, 241 (1996).

[26] G. Dvali, Q. Shafi, and R. Schaefer, Phys. Rev. Lett. 73, 1886 (1994).

[27] A.D. Linde, Phys. Lett. 129B, 177 (1983); H. Murayama, H. Suzuki, and T. Yanagida, Phys. Rev. D 50, 2356 (1994); M. Kawasaki, M. Yamaguchi, and T. Yanagida, Phys. Rev. Lett. 85, 3572 (2000).

[28] T. Moroi and H. Murayama, Phys. Lett. B 553, 126 (2003).

[29] M. Postma, Phys. Rev. D 67, 063518 (2003). 